\title{
Preface
}

\section{The Value of a Healthy Debate}

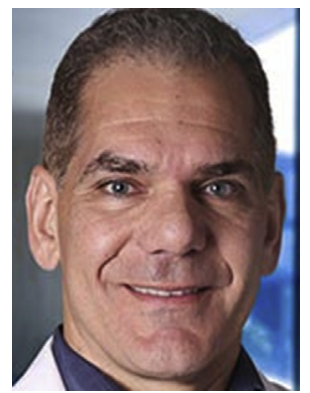

Emile G. Daoud, MD, FACC, FHRS

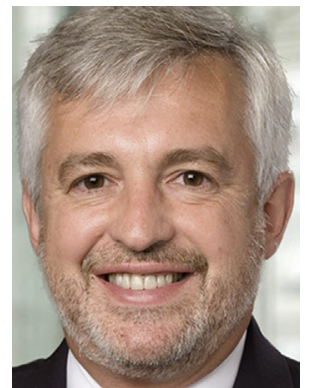

Raul Weiss, MD, FACC, FAHA, FHRS

Editors

Electrophysiology is a technical science. Measurements are made in milliseconds, and mapping is within 2 millimeters to selectively ablate only a small region of interest. With all this focus on precision, even experts in the field often have heated debates. These debates are often highlights at national convention meetings, and, in part, this is the motivation for the current issue of Cardiac Electrophysiology Clinics. In this issue, the goal of each article is to present a strong opinion in support of a particular method of patient management based on the author's interpretation of the literature. Some topics are presented as two articles: one article offering the PRO opinion and the second article offering the CON opinion. Other articles offer a detailed review of a topic as well as present the expert's opinion.

Regardless of the reader's personal preference for managing each individual patient scenario, these articles provide an excellent review of the literature coupled with extensive clinical experience, representing the merger of the science and the art of electrophysiology. Also, authors were invited to offer opinions from varied regions of the world, and topics were selected to address more than merely a single clinical scenario. Some of the articles address longterm risks and the medical ethics of clinical care.
Controversies exist when trying to apply the results of multicenter, randomized trials to a unique patient. This issue was designed to create difficult clinical scenarios to spark debate and passion for each author to support their point of view. These articles are relevant, entertaining, and educational.

\section{Emile G. Daoud, MD, FACC, FHRS Wexner Medical Center at The Ohio State University \\ 473 West 12th Avenue DHLRI, Suite 200 Columbus, $\mathrm{OH}$ 432210, USA}

Raul Weiss, MD, FACC, FAHA, FHRS Cardiac Electrophysiology Fellowship Program Wexner Medical Center at The Ohio State University 473 West 12th Avenue DHLRI, Suite 200 Columbus, OH 432210, USA

E-mail addresses: emile.daoud@osumc.edu (E.G. Daoud) Raul.Weiss@osumc.edu (R. Weiss) 